\title{
Survey of applications of discrete vortex method in civil engineering
}

\author{
Tomasz Nowicki \\ Lublin University of Technology, Faculty of Civil Engineering and Architecture, \\ Department of Structural Mechanics, e-mail: t.j.nowicki@pollub.pl
}

\begin{abstract}
This paper includes an introduction to Discrete Vortex Method (DVM) and its comparison with other methods of computer fluid dynamics. In its second part it focuses on the most important applications of DVM from the civil engineering point of view. Some examples known form literature are presented theoretical as well as experimental.
\end{abstract}

Key words: Discrete Vortex Method, Navier-Stokes equation.

\section{Introduction to Discrete Vortex Method}

Discrete Vortex Method (DVM) is one of numerical methods used for computer simulation of turbulent fluid flows. The origin of DVM is dated to the 1930s. However fast development in vortex methods started in 1980s preceded by progress in computer technology. Since then DVM has been successfully applied to many scientific and engineering problems. It has been proved to be useful in fluid mechanics, plasma physics, particle dynamics and fluid penetration in porous media phenomena. For 30 years DVM has been successfully used in civil engineering. However it is still a young method and it is not known widely among civil engineers. A pioneer computer system based on DVM algorithms is being developed at the Department of Structural Mechanics of Faculty of Civil Engineering with cooperation with Wind Engineering Laboratory at Cracow University of Technology.

\subsection{Elementary mathematical equations}

DVM is numerical method developed for solving the Navier-Stokes equation $(\mathrm{N}-\mathrm{S})$ based on Lagrangian model of particle tracing. In DVM the N-S equation is solved by direct simulation of physics phenomena. A finite mesh known from finite element method or finite volume method is not used in DVM.

Assuming a homogeneous fluid with constant viscosity we can write the N-S equation in the form (1):

$$
\frac{\partial \mathbf{u}}{\partial t}+(\mathbf{u} \cdot \nabla) \mathbf{u}=-\nabla p+\frac{1}{\operatorname{Re}} \Delta \mathbf{u},
$$

where: $\mathbf{u}$ - velocity field, $\mathbf{p}$ - pressure field, $R e$ - Reynolds number, $t$ - time.

The equation (1) is decomposed by calculation the rotation of vector $\mathbf{u}$, which gives the vorticity transport equation (2) 


$$
\frac{\partial \boldsymbol{\omega}}{\partial t}+(\mathbf{u} \cdot \nabla) \boldsymbol{\omega}=\frac{1}{\operatorname{Re}} \Delta \boldsymbol{\omega},
$$

where: $\boldsymbol{\omega}=\nabla \times \mathbf{u}$ is a voricity field of the flow.

The equation (2) is composed with two parts: advection (3) and diffusion (4) part.

$$
\begin{aligned}
& \frac{\partial \boldsymbol{\omega}}{\partial t}+(\mathbf{u} \cdot \nabla) \boldsymbol{\omega}=0, \\
& \frac{\partial \boldsymbol{\omega}}{\partial t}=\frac{1}{\operatorname{Re}} \Delta \boldsymbol{\omega} .
\end{aligned}
$$

The separation lets us treat a fluid flow as two simultaneously phenomena: advection and diffusion and it is knows as the Split Algorithm. It is the core idea for computer simulation of a fluid flow in DVM.

In DVM a continuous vorticity field is replaced with a discrete finite set of vortexes, that is called a vortex cloud. A discrete vortex is characterized with its strength and position in a calculation domain. The strength of a vortex is given by a formula (5). The equation (5) can be used for discretization the velocity field.

$$
\Gamma=\int_{L} \mathbf{u} \cdot \mathbf{d r}=\int_{S} \boldsymbol{\omega} \cdot \mathbf{n} d S,
$$

where: $\Gamma$ - strength of a vortex, $L$ - oriented closed curve with an element dr, $S$ area enclosed by the curve with an element $\mathbf{n}$.

The discrete vortex set let us reconstruct the velocity field on the basis of a formula (6).

$$
\mathbf{u}(\mathbf{x})=\sum_{i=1}^{N} \Gamma_{i} \mathbf{K}\left(\mathbf{x}-\mathbf{x}_{i}\right)
$$

where: $\mathbf{x}=(x, y)$ - position in two dimensional space, $\mathbf{K}$ - Biot-Savart kernel, $\mathrm{N}$ number of discrete elements.

The pressure field can be calculated with the Poisson equation (7)

$$
\Delta p=-\frac{\partial}{\partial t} \nabla \cdot \mathbf{u}-\nabla \cdot(\nabla \cdot \mathbf{u}) \mathbf{u} .
$$

Therefore the vortex cloud completely describes the flow field in an arbitrary point and time moment.

\subsection{Computer simulation process}

Computer simulation of advection of a fluid flow composed of discrete elements (Fig. 1) consists in calculating velocities for every vortex element according to the equation (6) (Fig. 2) and moving the discrete elements to new positions on the basis of calculated velocities and chosen time step. In order to reduce a numerical error at least a second order procedure should be applied (8). 


$$
\begin{aligned}
& \mathbf{x}_{i}^{n+0.5}=\mathbf{x}_{i}^{n}+\mathbf{u}_{i}^{n} \cdot \Delta t \\
& \mathbf{x}_{i}^{n+1}=\mathbf{x}_{i}^{n}+\frac{1}{2}\left(\mathbf{u}_{i}^{n}+\mathbf{u}_{i}^{n+0.5}\right) \cdot \Delta t
\end{aligned}
$$

where: $\Delta t$ - time step, $i-$ a number of a vortex, $n, n+1$ - number of a simulation step, $n+0,5$ - intermediate simulation step

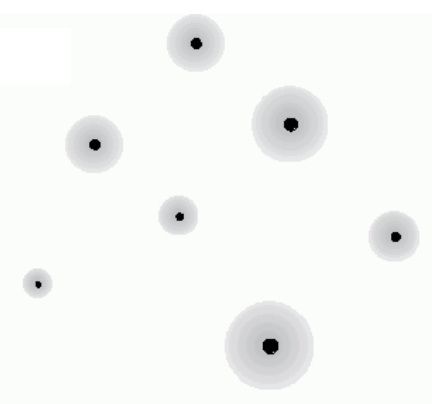

Fig. 1. Discrete vortex set.

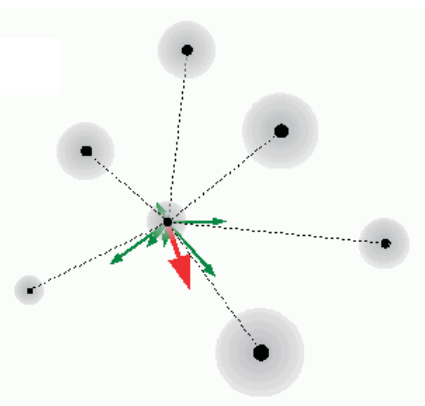

Fig. 2. Resultant velocity determined for a chosen vortex particle

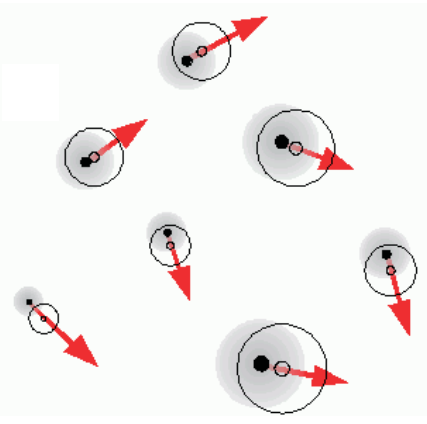

Fig. 3. Translation of vortex particles.

Computer simulation of diffusion of a fluid can be realized in a few alternative ways. The most proper for flows characterized with high Reynolds Number it the Brownian motion methods that results form the solution of equation (4). In the diffusion step of computer simulation every vortex element is moved from its position according to formula (9): 


$$
\begin{aligned}
& \mathbf{x}_{i}^{n+1}=\mathbf{x}_{i}^{n}+\xi_{i} \\
& p(|\xi|)=\frac{1}{4 \pi v \Delta t} e^{\frac{-|\xi|^{2}}{4 v \Delta t}},
\end{aligned}
$$

where: $\nu$ - kinematic viscosity; $\xi$ - displacement which module is a Gaussian random variable and angle a random variable with a uniform distribution.

During simulation process boundary condition must be fulfilled: no-throughflow (10) and no-slip (11) (Fig. 4).

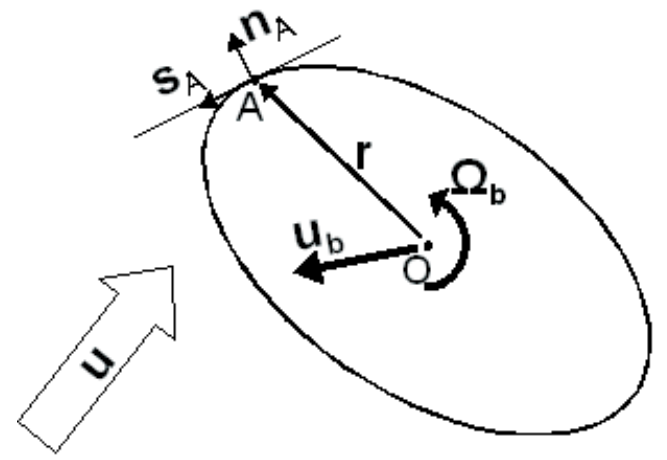

Fig. 4. Boundary conditions.

$$
\begin{aligned}
& \mathbf{u} \cdot \mathbf{n}_{A}=\left(\mathbf{u}_{b}+\boldsymbol{\Omega}_{b} \times \mathbf{r}\right) \cdot \mathbf{n}_{A}, \\
& \mathbf{u} \cdot \mathbf{s}_{A}=\left(\mathbf{u}_{b}+\boldsymbol{\Omega}_{b} \times \mathbf{r}\right) \cdot \mathbf{s}_{A},
\end{aligned}
$$

where: $\mathbf{u}$ - velocity of the fluid flow, $\mathbf{u}_{\mathrm{b}}$ - velocity of a body, angular velocity of the body, $\mathbf{n}_{\mathrm{A}}, \mathbf{s}_{\mathrm{A}}$ - unit vectors in point $\mathrm{A}$.

The no-flow-through conditions says that the fluid does not penetrate the body while the no-slip condition signifies that the fluid sticks to the body's surface. Despite their simplicity realization of the boundary condition pose the key problem in DVM. It results from the fact that the conditions are formulated for the velocity field but they must be realized in terms of discrete vorticity.

\subsection{Discrete Vortex Method in comparison with other methods of computational fluid dynamics}

Discrete Vortex Method is an alternative to mesh based methods used for solving Navier-Stokes equation. If the most important methods such as Finite Difference Method (FDM), Finite Element Method (FEM) and Finite Volume Method (FVM) are based on an arbitrary chosen mesh and require adding extra simplified models of turbulence such as Reynolds Average Navier-Stokes (RANS) or Large Eddy Simulation (LES). The DVM does not make use of a calculation mesh and simulates the phenomenon of turbulence in direct way. The lack of mesh makes the method very attractive for computer simulation of turbulent flows because the vortex elements are free to propagate in a computational domain. The freedom of 
propagation enables the vortexes to shape the phenomenon of turbulence during direct simulation process.

The attractiveness of the Discrete Vortex Method is balanced with its computational cost which is much higher in comparison to FDM, FEM or FVM. For $n$ vortex elements mutual interactions between them entails the need to perform $n^{2}$ calculations of velocities for one simulation step, which is described in literature as the $n$-body problem. For a typical engineering task there is a need to use about $5 \cdot 10^{4}$ elements and perform on them approximately $10^{5}$ calculation steps, which makes in total $\left(5 \cdot 10^{4}\right)^{2} \cdot 10^{5}=10^{13}$ single numerical calculations. The estimated computation cost is too high for contemporary computer hardware. The solution for the situation is using fast $n$-body simulators. The simulators divide the simulation domain into separated regions. Interactions within one cell are calculated in direct way but the longer distance interactions are calculated from a group to a group rather then from a vortex to a vortex. The avoidance of direct interactions works in reduction of calculations complexity from $O\left(n^{2}\right)$ to $O(n \cdot \ln (n))$, which results in reduction simulation times form years to hours.

\section{Survey of application of Discrete Vortex Method in civil engineering}

\subsection{Parameters of flow over a building}

The cited example concerns a real building included in Texas Tech University campus [2]. The analyzed object was cubicoid in shape of dimensions $9.1 \mathrm{~m}-13.5 \mathrm{~m}$ in plane and $4 \mathrm{~m}$ in height. The building was equipped with measurement utilities allowing monitoring parameters of wind flow over it. It was localized in a flat open area far from other buildings. A power law boundary-layer velocity wind profile with an exponent $\alpha=0.18$ and a mean speed at the building height $8.6 \mathrm{~m} / \mathrm{sec}$ was assumed. The wind flow had an angle-of-attack $90^{\circ}$ with the reference to the longer wall of the object. The problem was simplified to a two dimensional flow case and aeroelastic effects were omitted. The numerical simulation was performed for the Reynold's number $\mathrm{Re}=2.3 \cdot 10^{6}$. The number of vortex particles varies form 12000 to 24000 during the computational process. Numerical results were compared with experimental data. Selected results have been presented at figures form 5 to 7 . Figures 5 and 6 concerns the same moment of time.

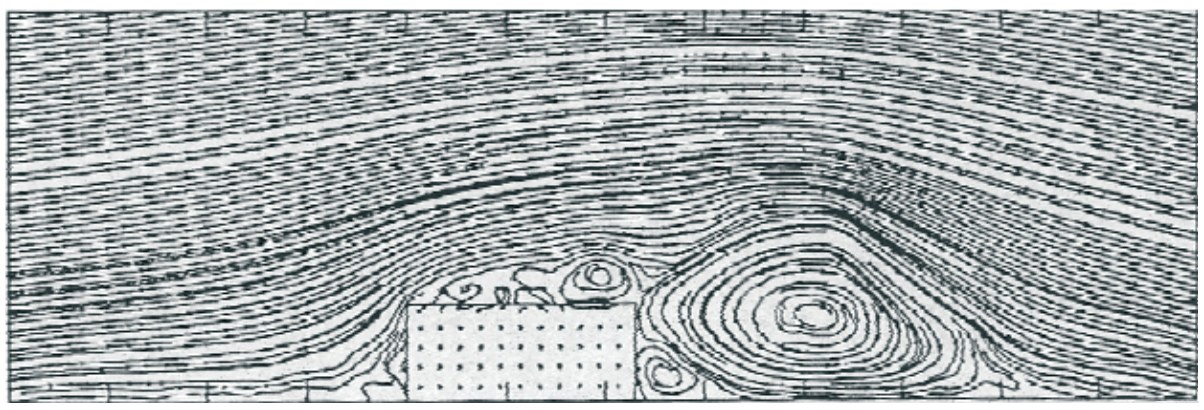

Fig. 5. Streamlines of the flow over Texas Tech building (visualization) [2]. 


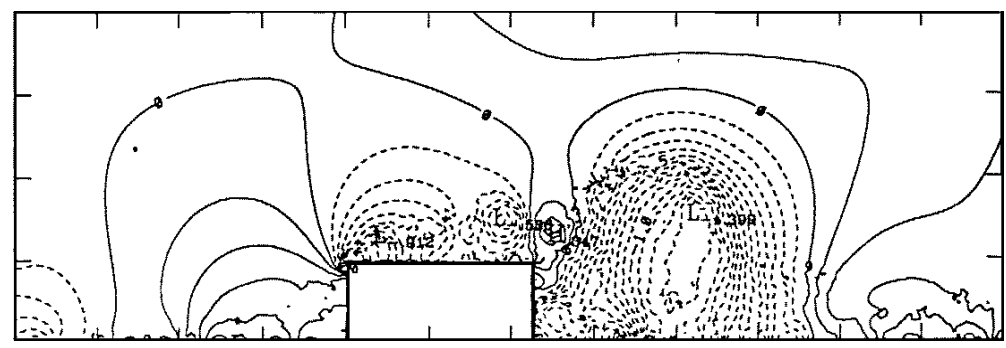

Fig. 6. Pressure field over Texas Tech building (visualization) [2].

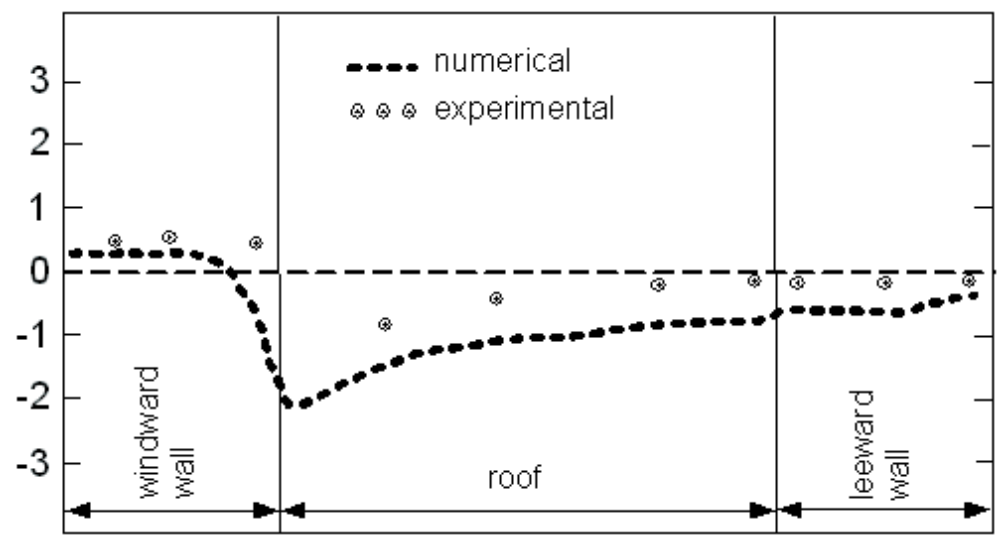

Fig. 7. Mean pressure coefficients on Texas Tech Building's walls (dimensionless) [2].

The quoted example shows good conformity between numerical simulation results and experimental data. The most important achievement is the shape of the line obtained from computer simulations. It shows that DVM algorithms are able to model the phenomena of turbulence that occurs near edges of a body like the place where wall connect with the roof. The discrepancy between experiment and calculations, that can be noticed in the Fig. 7, comes from the fact that the building was not long enough (the length to depth ratio was equal 1.5) to treat the flow as two dimensional and the influences of walls parallel to the flow should not have been neglected and three dimensional analysis of the flow would have been more proper in this case. However, the differences are small and do not disqualify the results with a civil engineer's point of view.

Researches presented in [2] show a wide area of applications in civil engineering practice. It is possible to estimate wind loads on building. Both the static mean and dynamic values can be obtained. The results are of high importance for a structural engineering whose role is to ensure safety of a building structure. Except forces acting on a building's planes it is easy in DVM to obtain physical parameters of flow in an arbitrary point within a simulation domain. It is possible to follow changes of pressure or velocity of fluid at a chosen point in space. Therefore trouble spots such as air vents or outlets or chimneys can be tested to possibility of reverse thrusts. Visualization of air flow (Fig. 5) enables to indicate areas with deteriorated wind comfort. All of the mentioned analysis can be made at the design stage as well as for an existing buildings. Moreover complicated geometry of an object is not an obstacle for DVM, because it does not make use of calculation mesh. 


\subsection{Determination of aerodynamic characteristics}

Researches presented in [3] concern determination coefficients of aerodynamic resistance: drag $C_{X}$, lift $C_{Y}$ and moment $C_{M}$. Square and rectangular sections (Fig. 8) were analyzed against wind action with a different angle of incidence. The shape ratio $L / B$ varies from $1 / 4$ to 3 and the angle of incidence $\alpha$ from $45^{\circ}$ to $90^{\circ}$. Two dimensional flow field was assumed. During the analysis Reynolds number came to $2 \cdot 10^{4}$.

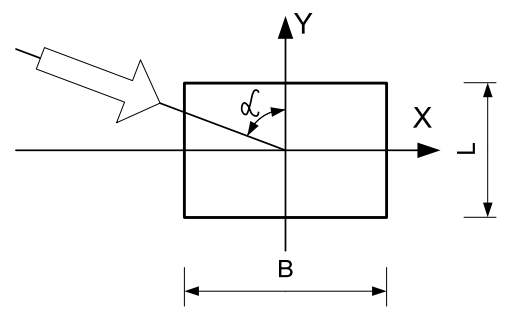

Fig. 8. The shape being analyzed.

Figures from 9 to 11 present calculation results confronted against experimental data for the case of $L / B=1$.

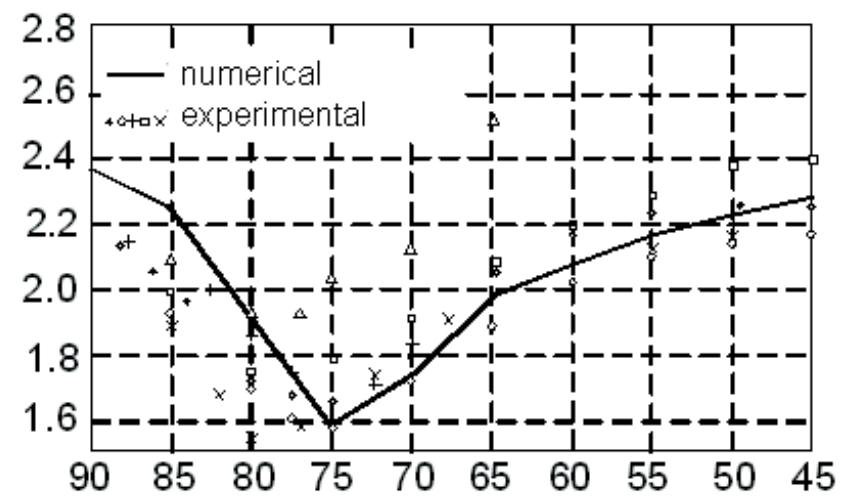

Fig. 9. Mean drag coefficient vs. angle of incidence (dimensionless) [3].

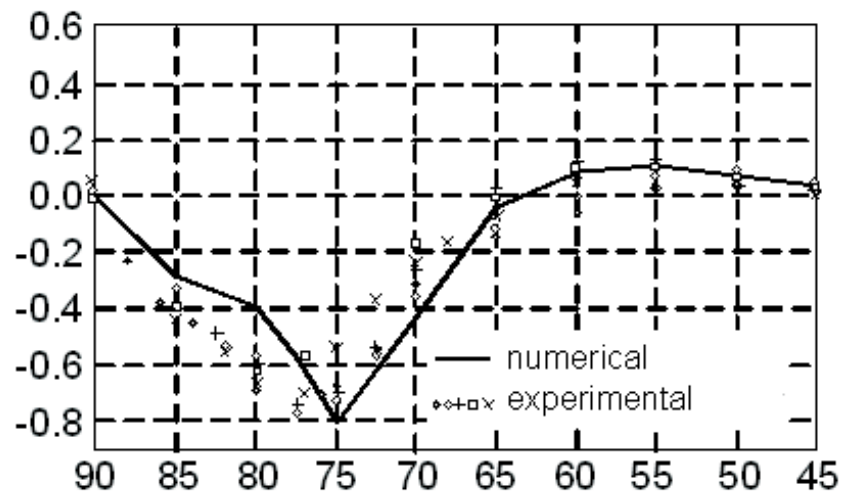

Fig. 10. Mean lift coefficient vs. angle of incidence (dimensionless) [3]. 


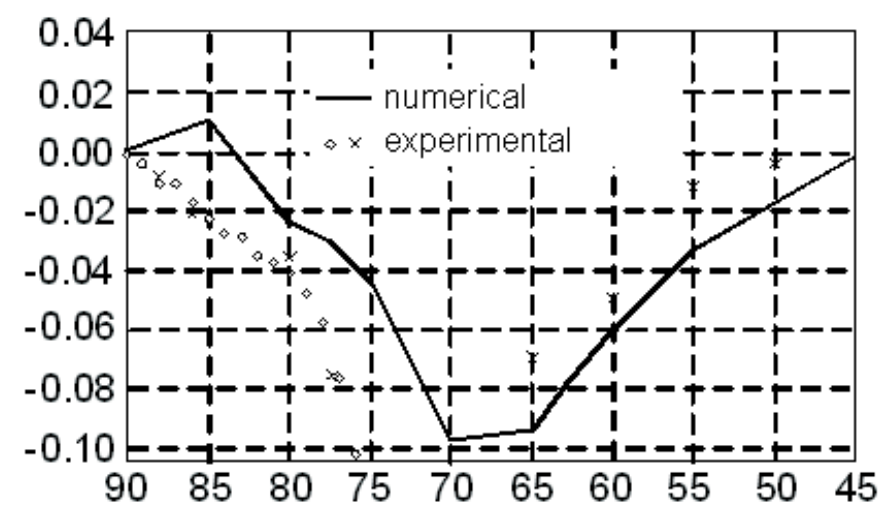

Fig. 11. Mean moment coefficient vs. angle of incidence (dimensionless) [3].

Quoted results obtained from DVM analysis may confirm in the conviction that the method can be successfully applied to determine coefficients of aerodynamic resistant. The characteristic are needed for civil engineers while designing slender structures for which wind action is the most important load. It concerns cable stayed masts, towers, suspended or cable stayed bridges. The coefficients are evaluated experimentally in the long and expensive process. Any changes in a project of a structure involves repeating of the process. DVM algorithms enables to shorten the time of designing and reduce costs.

\subsection{Prediction of dynamic response of a structure with aeroelasticity effects}

Undeniable superiority to other method to computational fluid dynamic DVM presents in analysis including aeroelastic interaction. An example of such simulation one can find in [4], where the authors presents their results of dynamic response of a bridge glider placed in a turbulent wind flow. They used DVM to examine aeroelastic stability of Tacoma Narrow Bridge and compared the results with historical experimental data. Tacoma Narrow Bridge collapsed $7^{\text {th }}$ November 1940 as a result of intense flutter caused by wind blowing with mean speed $19 \mathrm{~m} / \mathrm{sec}$. According to historical resources the angle of bridge deck reached value of $30^{\circ}$ just before the catastrophe.

Numerical simulations presented in [4] concerned only a deck of the bridge (Fig. 12). Because flutter occurs in center area of a bridge and bridge spans are at least a few dozen times longer then their width limitation simplification to two dimensional analysis are justified and does not introduce big errors.

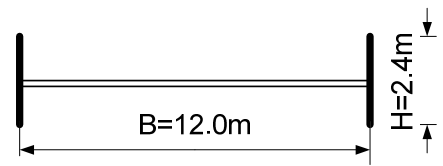

Fig. 12. Dimensions of Tacoma Narrows Bridge's girder.

Computer calculations reconstructed the phenomena that happened in 1940. Numerical experiments conducted for wind speed in a range form $5 \mathrm{~m} / \mathrm{sec}$ to $20 \mathrm{~m} / \mathrm{sec}$. 
They confirmed that the critical speed for flutter was $19 \mathrm{~m} / \mathrm{sec}$. Visualization of the results showed that the amplitude of periodic torsions came to $30^{\circ}$ (Fig. 13). Vertical displacement are presented in Fig. 14.

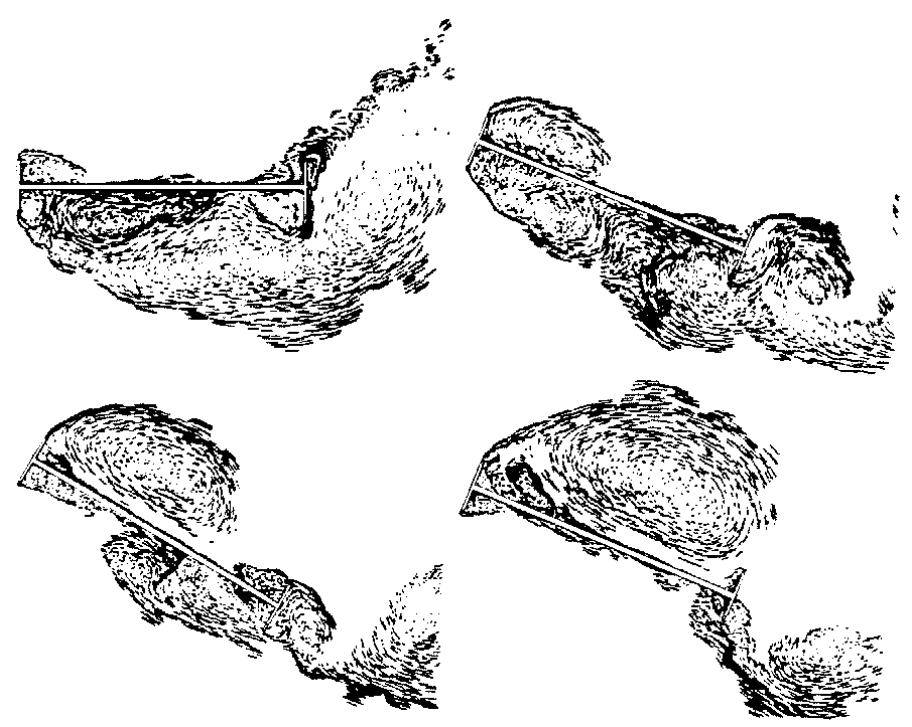

Fig. 13. Torsional flatter of the girder [4].

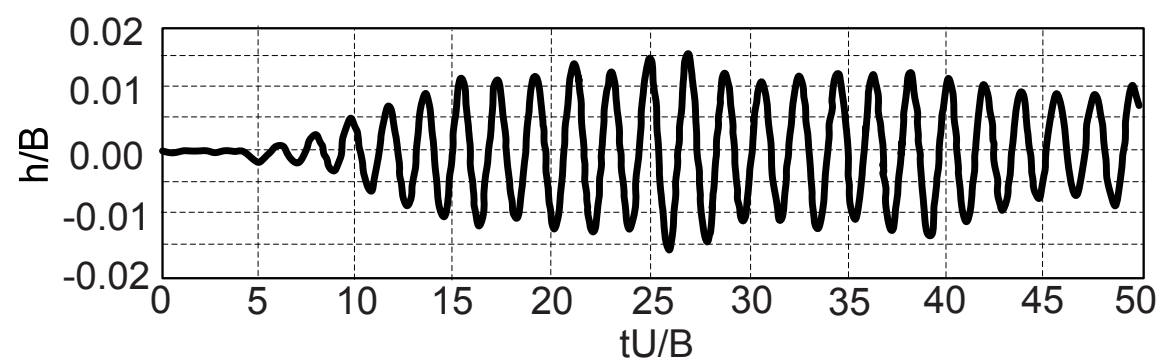

Fig. 14. Dimensionless vertical displacement of the girder vs. dimensionless time [4], where $h-$ displacement of o bridge, $B$ - width of the deck, $t$ - time, $U$ - wind speed.

The convergence between numerical results and known from history catastrophe encourage to state that DVM analysis should always be involved in the process of designing suspended or cable stayed bridges. It enables to discover and eliminate the most prone to aeroelastic instability structure variants. Since the cost of computer simulations is much more lower then the cost of experimental test it is possible to examine more variants in shorter time, choose the best one and test it in a wind tunnel laboratory.

The quoted example concerns a bridge structure but the aeroelastic analysis can be performed to any kind of building structure such as masts, towers, single cables, etc.

It is worth mentioning that aeroelastic simulation are very difficult to carried out using mesh based methods (FEM, FVM) because a movement of a body in a flow require remeshing at every calculation step. 


\section{Summary}

Taking into consideration fast development in computer technology the Discrete Vortex Method can be treated as a very promising and versatile tool of wind engineering. Computer programs based on DVM algorithms have proofed to be useful in a process of designing structures for which wind dynamic load is the most important one. Computer programs based on DVM algorithms are sure to become a regular engineering tool in the same way computer programs based on FEM or FVM are used nowadays. They will be used to estimate wind load on buildings, their dynamic response to wind and for wind comfort assessment.

However, one must state that DVM is a relatively young calculating method and still requires intensive scientific researches before it become a regular design engineering tool. At present it can be considered a valuable aid for traditional methods of wind engineering.

\section{References}

[1] Cottet G.H., Koumoutsakos P.D., Vortex Method: Theory and Practice, Cambridge University Press, 2000.

[2] Turkiyyah G., Reed D., Yang J., Fast vortex methods for predicting wind - induced pressures on buildings, Journal of Wind Engineering and Industrial Aerodynamics 58, 1995.

[3] Taylor I., Vezza M., Prediction of unsteady flow around square and rectangular section cylinders using a discrete vortex method, Journal of Wind Engineering and Industrial Aerodynamics 82, 1999.

[4] Larsen A., Walther J.H., Aeroelastic analysis of bridge girder sections based on discrete vortex simulation, Journal of Wind Engineering and Industrial Aerodynamics 67\&68, 1997. 